\title{
Postpartum Depression and Access to Mental Healthcare among Migrant South Asian Women in Canada: A Literature Review
}

\author{
Samar Khan \\ Faculty of Humanities, McMaster University, Canada
}

\begin{abstract}
Although there has been an increase in research on postpartum depression (PPD) among migrant Asian women, there is particularly limited attention given to migrant South Asian women. This is a significant limitation considering the rapidly changing demographics in Canada. Migrant South Asian women face a plethora of unique challenges, which affects their mental health and further prevents them from receiving adequate and equitable mental healthcare services. For migrant South Asian women, many of these stressors are especially amalgamated in the vulnerable postpartum period, resulting in variances of postpartum mood disorders such as postpartum blues, non-psychotic postpartum depression, and in more severe cases, postpartum psychosis. While cultural values and rituals may be protective in curbing PPD symptoms in some instances, in other cases, however, they exacerbate and perhaps even worsen PPD. The objective of this literature review is to provide a basic understanding of postpartum depression among the migrant South Asian community in Canada. It aims to answer questions pertaining to the types of postpartum mood disorders that women can potentially suffer from, its risk factors, its prevalence among migrant women, as well as the socio-cultural and gender-related barriers to attaining access to mental healthcare services and treatments for PPD in Canada. Around 40 documents were examined, summarized and synthesized for this literature review. These included scholarly journal articles, non-scholarly articles, health-related websites, position papers, and medical journals.
\end{abstract}

Keywords Postpartum Depression, Migrant, Women, Mental Health, South Asian

\section{Introduction}

Over the past several decades, the number of migrants arriving to Canada from non-European countries within South/East/Central Asia, the Middle East and Africa has increased significantly (O'Mahony and Donnelly, 2010). According to Citizenship and Immigration Canada (CIC) (2003), a total of 221,340 immigrants and refugees entered Canada from various countries around the world. A more recent Statistics Canada (2010) report indicated that only within the past decade, Canada has welcomed over two million newcomers from around the world. Among the most prominent of these countries are India and Pakistan, respectively (Agarwal-Narale, 2005). This high number of migrants arriving from places within South Asia indicates that South Asians are among the fastest growing populations in Canada. A significant number of this population includes childbearing families, which hence poses a challenge for many healthcare providers in especially trying to meet the needs of migrant South Asian women, who are struggling to not only adapt to their new environment, but to also cope with mental illnesses such as postpartum depression. Katz \& Gagnon (2002) and Kinnon (1999) argue that migrant women's vulnerability to mental illnesses is further exacerbated when they are provided with less than optimal mental healthcare services, especially shortly after giving birth due to cultural, linguistic and socio-economic barriers that shape and contribute to their postpartum experiences (Sword, Watt and Kreuger, 2006).

Further, migrant South Asian women are one such population that experiences a plethora of health inequities. This statement is supported by MacDonell, Dastjerdi, Bokore, and Khanlou (2012, p.1), as they argue that migrant South Asian women "are not a homogenous group, having different contextual supports and challenges as for the most part their lives are represented in mainstream discourse as a homogenous population 'in need', in terms of their risks for disease, lack of access to culturally relevant services, and thus their burden on the health system." However, at the same time, it is imperative to understand that migrant South Asian women's vulnerabilities are contextualized in terms 
of their status as 'racialized' women who face barriers pertaining to cultural discrimination and devaluation of their health concerns (Armstrong, Boscoe, and Clow, 2003).

This study aims to provide insight into the mental health promotion context of settlement issues for migrant women of South Asian descent, living in Canada, whose voices are often unheard as English is not their first language. Although there is adequate research available concerning the mental health promotion of migrant women in Canada, there is however very little research available that specifically addresses the postpartum mental health experiences of South Asian women in Canada. There is very minimal understanding of the social and cultural barriers these women face in trying to access mental healthcare services when coping with PPD, which in turn affects the quality of care they receive as it is often limited by a lack of care or acknowledgement among healthcare providers.

Although the Canadian healthcare system is known for its high quality prenatal care and birthing facilities, the area where it falls significantly short is when it comes to addressing emotional, psychological, and cultural/linguistic needs. Hence, there appears to be a lack of sufficient research on PPD among migrant South Asian women that sheds light on the women's perspectives of their social support needs. Yet, the few studies that are available suggest that the psychological health of migrant South Asian women is compromised not only due to the reason of having to cope with motherhood, but also due to feelings of loneliness derived from isolation and lack of social support (Sword et al., 2006). Minas et al. (2013) argues that, "the support of women's mental health needs to be the 'core businesses' of services that are responsive, accessible, and culturally appropriate in order to meet the challenges presented by women of diverse cultural and linguistic backgrounds."

\section{Research and Discussion}

\section{Perception of Postpartum Depression among South Asians}

Postpartum depression is a mental illness that is experienced by women all over the world. Some are fortunate enough to live in places where this illness is recognized for its austerity and are hence provided the required medical attention and care to treat it. There are other women, however, who are not as fortunate simply because the condition is either not recognized nor acknowledged as a 'mental illness' in the first place. For the most part, these are women who either hail from or belong to South Asian communities. There is much stigma attached to understanding the concept of mental illness. This stigma hence has very unfavourable effects, especially on South Asian women suffering from postpartum depression, because they are more than often not taken seriously. South Asian women are expected to quickly adapt to their roles as mothers soon after the birth of a child, without any nakhrey (tantrums). Any woman who complains or shows any signs of distress is automatically deemed as pagal (insane), which hence becomes a major deterrent in their ability to seek professional medical help.

According to Kakar (1991), social stigma and shame in the South Asian culture is often associated with the suffering of any kind of mental illness, and is not only limited to postpartum depression (Agarwal-Narale, 2005). Similarly, Tewary (2005) stated that mental illnesses in the South Asian culture is perceived to be a non-issue and that they are actually self-inflicted by the women themselves in order to "gain attention" (Sanghera, 2014). Hence, when it comes to culture, many South Asians believe and have even convinced themselves that any mental illness is not an illness at all, and that there is no need to seek any medical help. However, if it were determined that one is suffering from a mental illness, rather than providing the individuals with the means and access to medical help, they are first forced to practice self-discipline to rid themselves of the mental illness. In other cases, the family of the individual will insist that they help the patient out, before seeking any sort of medical treatment (Sanghera, 2014). The concept of mental illness is either not recognized or simply dismissed by South Asians as they believe that it is impossible to suffer from any sort of mental illness when most South Asians are known to have close knit relationships with their families, take good care of their health, and engage in religious/spiritual activities (Agarwal-Narale, 2005).

\section{Socio-cultural Barriers}

\section{Language Problems}

Lack of knowledge or fluency in English is undoubtedly one of the biggest impediments for migrant South Asian women when trying to gain access to mental healthcare services in Canada. Due to this language barrier, women are unaware about where and how to get the necessary information in order to seek help for postpartum depression. Although some hospitals and clinics provide translation and interpretation services, it is usually not ideal due to lack of availability and it can also be quite inefficient and time-consuming. Usually the expectation is that these women would have some practical knowledge of English in order to communicate their problems to their respective psychologists or healthcare providers, rather than have a translator relay all or most of the information to the psychologist/healthcare provider. This is supported by a study conducted by Teng, Robertson Blackmore, and Stewart (2007), which examined healthcare workers' perceptions of barriers to care by immigrant women who were suffering from postpartum depression. One of the key informants who were interviewed stated the following:

Greta [psychologist]: 'in my experience, trying to communicate with a woman suffering from PPD through a translator [sometimes] feels like...an 
exercise in futility. (Teng et al., 2007, p. 95-96)

\section{Social Isolation}

One of the major barriers that many migrant South Asian women face when they migrate to Canada is that of social isolation. This is because they come from a culture that deeply values family life and living in extended families. As a matter of fact, among most traditional South Asians, living in a joint family is considered ideal and favourable. Further, for many South Asian women, childbirth is seen as a family experience, where family members such as the mother or mother-in-law provide both emotional and physical support to ensure the woman has a smooth and healthy pregnancy and birth (Grewal, Bhagat, and Balneaves, 2008). It needs to be understood that this family support is not limited to women who are living 'back home', but to also those living in Canada, and who are fortunate enough to have their families living with them to provide them with the support they need. However, not all migrant South Asian women are fortunate enough to migrate to Canada, and bring along their families. It is practically impossible, as it may take up to several years for refugee women, especially, to be reunited with their families again. As a result, most of these women arrive with only their husbands, and sometimes even alone, and if they are already pregnant, they fall at a high risk of suffering from postpartum depression due to lack of support and feeling socially isolated. This relates to a study conducted by Sanghera (2014), in which she conducted interviews with pregnant immigrant South Asian women who were suffering from symptoms of depression. One of her participants, who had migrated to Canada from India, stated the following:

I just miss my family in India (pause). We had quite a large family and here all day I am on my own, because husband is at work and that's why...Those who come new here they should have some sort of family here close around them... like for one year everyone should feel like there is someone close to them and then you will like it here because for me it is extremely hard. (Sanghera, 2014, p. 79)

This is further supported by a focus group study that was conducted by Ahmad et al. (2004) which revealed that the loss of social support was viewed among immigrant South Asian women as one of the biggest stressors to suffering from mental health (Sanghera, 2014). Additionally, a study conducted in Toronto by Teng et al. (2007), revealed that other barriers included obligations to family and marriage, lack of spousal support and validation, increased dependency on the husband, and unsupportive mothers-in-law (Sanghera, 2014).

\section{Financial/Income Struggles}

One of the main issues migrant South Asian women face upon their initial arrival to Canada is economic and financial burdens. Many of these women are also financially dependent upon their husbands, who may be forced to work long hours at hard labour jobs. This is due to the reason that their degrees from their respective countries may not be recognized/accepted, or because they are not educated and may lack English language skills, which thus makes it difficult for them to find proper jobs $\left(\mathrm{O}^{\prime}\right.$ Mahony and Donnelly, 2010). In an important study carried out by Sanghera (2014), one of her participants -- an immigrant woman from South Asia -- stated the following:

All day I am on my own, because husband is at work and that's why... he does driving, drives a taxi and so sometimes can work 14 hours. (Sanghera, 2014, p. 89)

This result in lack of spousal support for the new mothers, which in turn leads to financial issues and the mother hence suffers from postpartum depression. According to Miszkurka et al. (2010), a low socio-economic status leads to much higher depressive symptoms among migrant South Asian women (Sanghera, 2014).

\section{Gendered Roles and Expectations}

Understanding the concept of gender roles and expectations in the South Asian culture is important in order to understand how migrant women experience postpartum depression. Families in the South Asian culture are primarily patriarchal, where according to Mehrotra and Calasanti (2010) men (especially elders) hold the most power, whereas the daughters-in-law hold the least amount of power. Hence, due to the patriarchal setup of families and the culture as a whole, women's roles are limited to the household, which contributes to worsening their mental stress and anxieties, thus increasing the risk of suffering from postpartum depression (Grewal, Bottorff, and Hilton, 2005).

Further, when South Asian women get married, they are expected to live with the husband's family, in their home, and are further required to take on roles and responsibilities such as cooking, cleaning, and other excessive amounts of domestic work at large. Hence, the high demands and responsibilities placed on South Asian women exacerbate their PPD experiences, making it impossible for them to seek any medical help. Additionally, when a woman is part of a strictly patriarchal household, where she is forced to comply with gendered roles and expectations and where her agency is limited, she is more prone to developing serious mental health issues that require immediate attention.

\section{Newborn Gender Preference}

Traditionally, in the South Asian culture, male children are greatly preferred over female children. This traditional notion comes from the belief that males are the ones who will carry on the family name. Although, among more educated and enlightened South Asians, the expectation to produce a male child is shifting away, some South Asian immigrant women still feel the pressure to have a male child, causing them much distress. According to Lynam 
(2000), women who do not produce a male child, while continuing to give birth to female children, fall at a much greater risk of suffering from postpartum depression, as the amount of stress that their families and in-laws place on them becomes greatly overwhelming (Sanghera, 2014).

Further, according to several studies conducted by Chandran et al. (2002), Patel et al. (2002), Rodrigues et al. (2003), and Savarimuthu et al. (2010), there appears to be a great desire for newborn males, and it is quite consistent in many South Asian studies related to postpartum depression, in which it is also considered to be a major stressor for mental illnesses to exacerbate (Sanghera, 2014). Failure to give birth to a male child further adds distress and depression in the form of domestic and marital violence, because blame is often placed on the woman for not being able to produce a male child.

\section{Inequities in Healthcare Access for Migrant South Asian Women}

\section{Racial Discrimination}

In addition to cultural issues such as language barriers and difficulties in comprehending the variety of mental healthcare services that are available for women who are suffering from PPD symptoms, there is also the issue of racial discrimination that exists, albeit subtle, in the Canadian healthcare system. In a qualitative research conducted by Agarwal-Narale (2005), in which she looked at the mental health of South Asian women in Canada post-migration, it was revealed that one of the participants she had interviewed -- a South Asian immigrant woman -reported insensitivity of staff towards her, which further resulted in long-waiting times before her needs for medical care were addressed. The reason that the participant felt she was being racially discriminated against was because she noticed situations in which other women, who appeared to be Caucasian, did not receive the same negative treatment as she did. As a matter of fact, they were often prioritized, and treated much more pleasantly. An example of racial discrimination that was quite apparent, as relayed by Agarwal-Narale, states as follows:

Many people hate Asians. They do not like Asians. And 1 know that here the English people even, they do not like Asians. I was in the same room in which there was a lady, she was French and I was Muslim. And the nurses, they didn't come to me. They went to her...When my baby cry, cried a lot, I called them, and there was a buzzer, you know, near the bed. I called them many times and they came after two hours. And the lady with me on the other bed, she called them and she pressed the buzzer, within ten minutes the nurse came. (Agarwal-Narale, 2005, p. 68)

Economic Inequities

Another major issue, along with racial discrimination, which serves as a barrier to accessing mental healthcare services for migrant South Asian women is economic inequities. In the qualitative research carried out by Agarwale-Narale, she noted that based on her interviews with South Asian women migrants, many of the healthcare providers were not sensitive to the economic or financial situations of these women. One of the participants that Agarwale-Narale interviewed mentioned how her physician was not sensitive to the fact that she could not afford high quality diapers for her newborn who had developed a severe form of diaper rash. Rather than considering the financial situation of the woman, the physician recommended that she invest in high-quality, expensive diapers and that she should change the diapers as frequently as possible. The physician did not bother to take the woman's financial situation into consideration, nor acknowledged the fact that the woman would not be able to afford the diapers (Agarwal-Narale, 2005).

Another issue was that of transportation. Many migrant South Asian women arrive to Canada, unable to afford to buy a car, much less drive. So, they rely heavily on public transportation. However, most of the clinics are far, and there are instances that some women cannot even afford to take public transportation, especially when they have to make regular visits to their healthcare providers. So, they resort to walking to and from the clinic/hospital, which can be both tiring and time-consuming. Hence, the location of healthcare services and the amount of time it takes serves as barriers to accessing proper healthcare, due to the lack of financial means (Agarwal-Narale, 2005).

\section{The Need for Culturally-sensitive Programs and Policies for Postpartum Care}

Considering the plethora of socio-cultural and healthcare accessibility barriers that migrant South Asian women face, it is imperative that more attention is drawn towards understanding the intersecting dimensions of language, culture, migration, and financial factors. Effective programs and policies need to be created in order to assist migrant South Asian women to become more comfortably integrated in Canada (Sanghera, 2014). Examples may include providing funds for improved and easily accessible English language and educational services for migrant women, so that they can overcome language barriers and gain more self-confidence (Sanghera, 2014). As the results of this reviw has shown thus far, overcoming language barriers will serve as a plausible solution for these women to overcome barriers to accessing mental healthcare services, as it will enhance their ability to interact with the healthcare system and verbally relay their problems and concerns. Also, along with providing more employment opportunities for South Asian women, following migration, it would also be a good idea to take their financial situations into consideration and provide them with more affordable housing so as to ease their financial burdens and make their transition to Canada easier (Sanghera, 2014). 
Furthermore, Canadian immigration policies need to be adjusted so that they can allow for migrant South Asian women in their postnatal/postpartum period to be reunited with their families, who want to visit and take care of them (Sanghera, 2014). Additionally, healthcare services need to be culturally sensitive, in that services should not only be provided in several South Asian languages, but should also be readily accessible to this population (Sanghera, 2014).

\section{Attributions of Coping Mechanisms, Treatment and Recovery}

\section{Cultural Values and Religious Rituals}

For some migrant South Asian women, cultural values and religious rituals and beliefs serve as a coping mechanism when faced with difficult times, and this includes suffering from postpartum depression. For these women, religion and spirituality especially serves as a positive and effective way in dealing with the troubles in their lives as well as overcoming health challenges. O'Mahony and Donnelly (2013) states that religious spirituality provides these women with a sense of comfort, especially in prayers and spiritual healing practices such as $R e i k{ }^{1}$, as well as different forms of yoga and meditation. As a matter of fact, for many migrant South Asian women, religious and spiritual healing holds high importance in the sense that it not only becomes a form of individual comfort, but also a source of community support (O'Mahony and Donnelly, 2013).

Hence, while struggling with the negative emotions of postpartum depression, migrant South Asian women's cultural values, religious and spiritual beliefs helps empowers them in a positive way, and further creates meaning and purpose in their lives.

\section{Forensic Mental Health and Psychiatry Services}

There are more than one type of postpartum mood disorders, and the most severe of them known as postpartum psychosis could lead to either suicide or filicide/infanticide. According to Milgrom et al. (2012), suicide rates appear to be the highest among migrant South Asian women, due to the many social and cultural barriers and challenges they face, especially when migrating to a foreign country. Since suicide has been identified as a concern within the female South Asian population in Canada, it is imperative that counseling psychologists attribute special attention and focus onto the psychological well-being of migrant South Asian women, both in the perinatal and postnatal period.

Further, migrant South Asian women who are at a high

${ }^{1}$ Reiki is a form of healing practice where the therapist, who is doing the healing, is known to channel his or her positive energy into their patients through touch. This healing process is understood to activate the natural healing processes of the patient's body, allowing for the restoration for physical, mental, and emotional well-being. risk of committing suicide (or have committed suicide, but somehow survived) could be a potential harm to their baby (especially if they have a history of harming their baby in the past), or if a woman has killed her child (committed infanticide), then she should be provided access to forensic mental health services. Forensic mental health services provide treatment and assessment for individuals who have either faced charges or criminal offences ("Forensic Mental Health," n.d.). Further, women should receive treatment through forensic psychiatry, which, "concerns the intersection of psychiatry and the law, including psycholegal questions about diagnosis, impairment, fitness to stand trial, criminal responsibility, and risk to offend" ("Forensic Mental Health," n.d.).

\section{Conclusion}

The findings of this literature review on postpartum depression have attempted to provide an understanding of the unique experiences of migrant South Asian women living in Canada. There is no doubt that migrating and settling in a new country can be a difficult and stressful experience, especially if some of these women led much more comfortable lifestyles in their 'home' countries. The downward mobility they experienced with regards to economic/financial struggles, language difficulties, racial discrimination, lack of social/family support, social isolation, and preference for a male child over a female child further intensified symptoms of postpartum depression and additionally affected their ability and confidence to seek medical help. Although perceptions around mental illnesses such as postpartum depression are linked to their cultural perceptions of mental health and illness, it was also discovered that these women have different coping strategies when dealing with psychological distress. These strategies were primarily personal /religious activities and confiding in family/community.

The findings also revealed that the lack of programs and policies to meet the needs of these women made it that much more difficult to access mental healthcare services. Hence, these findings confirmed not only an underutilization of mental healthcare services, due to the plethora of barriers and challenges facing immigrant and refugee South Asian women, but also highlighted the strategies, both cultural and religious, that these women prefer.

It is further anticipated that this literature review will be able to provide insight, awareness and understanding to academic researchers and healthcare practitioners about the many social and economic issues facing migrant South Asian women in Canada. It is also anticipated that both academic researchers and healthcare practitioners are able to identify, understand and respect the context of migrant South Asian women's experiences, and the ways in which those experiences affect their ability to cope with mental illnesses such as postpartum depression. Hence, healthcare 
practitioners and academic researchers need to be more open to diversity as well as be more culturally aware of the various lived experiences of South Asian women in Canada.

\section{REFERENCES}

Agarwal-Narale, T. (2005). Mental health of South Asian Women: Dialogues with recent immigrants on post-migration, help-seeking and coping strategies (Master's thesis, McGill University, Montreal, Canada). Retrieved from http://digitool.library.mcgill.ca/webclient/StreamGate?folder_id $=0$ \&dvs $=1450561338096 \sim 521$

Armstrong, P., Boscoe, M., and Clow, B. (2003). The Implications of the Final Report of the Commission on the Future of Health Care in Canada for Women, National Coordinating Group on Health Care Reform and Women and Canadian Women's Health Network, Manitoba, Canada.

Citizenship and Immigration Canada (2003). Facts and figures 2003. Retrieved from http://www.cic.gc.ca/english/pub/fac ts2003/index.html.

Forensic Mental Health (No date). What is Forensic Psychiatry and Forensic Mental Health? The Royal's Institute of Mental Health Research. Retrieved from:

http://www.theroyal.ca/research/areas-of-research/forensic-psychi atry/

Grewal, S., Bottorff, J. L., \& Hilton, B. A. (2005). The influence of family on immigrant South Asian women's health. Journal of Family Nursing, 11(3), 242-263.

Grewal, S. K., Bhagat, R., and Balneaves, L. G. (2008). Perinatal beliefs and practices of immigrant Punjabi women living in Canada. JOGNN: Journal of Obstetric, Gynecologic \& Neonatal Nursing, 37(3), 290-300.

MacDonell, J.A., Dastjerdi, M., Bokore, N., and Khanlou, N. (2012). Becoming resilient: promoting the mental health and well-being of immigrant women in a Canadian context. Nursing Research and Practice, 2012, 1-10.

Mehrotra, M., \& Calasanti, T. M. (2010). The family as a site for gendered ethnic identity work among Asian Indian immigrants. Journal of Family Issues, 31(6), 778-807.

Milgrom, J., Gemmill, A. W., Bilszta, J. L.,Patel, V., Ramasundarahettige, C., Vijayakumar, L., Thakur, J., Gajalakshmi, V., Gururaj, G., Suraweera, W. and Jha, P. (2012). Suicide mortality in India: a nationally representative survey. The Lancet, $379,2343--2351$.

Minas, H., Kakuma, R., San Too, L., Vayani, H., Orapeleng, S., Prasad-Ildes, R., Turner, G., Procter, N., Oehm, D. (2013). Mental health research and evaluation in multicultural Australia: developing a culture of inclusion. International Journal of Mental Health Syst., 7(1), 23.

O'Mahony, J. and Donnelly, T. (2010). Immigrant and refugee women's post-partum depression help-seeking experiences and access to care: a review an analysis of the literature. Journal of Psychiatric and Mental Health Nursing, 2010(17), 917-928.

O'Mahony, J.M., and Donnelly T.T. (2013). How does gender influence immigrant and refugee women's postpartum depression help-seeking experiences? Journal of Psychiatric and Mental Health Nursing, 2013(20), 714-725.

Sanghera, R. (2014). Exploring perinatal depression in South Asian immigrant women (Master's thesis, University of British Columbia, British Columbia, Canada). Retrieved from:

https://open.library.ubc.ca/cIRcle/collections/ubctheses/24/items/1 .0165899

Sword, W., Watt, S., and Kreuger, P. (2006). Postpartum Health, Service Needs, and Access to Care Experiences of Immigrant and Canadian-born Women. AWHONN, the Association of Women's Health, Obstetric and Neonatal Nurses, 35(6), 717-727.

Teng, L., Blackmore, E.R, and Stewart, D.E. (2007). Healthcare worker's perceptions of barriers to care by immigrant women with postpartum depression: an exploratory qualitative study. Archives of Women's Mental Health, 2007(10), 93-101. 